\title{
Quantum Chiral Higher Spin Gravity
}

\author{
Evgeny Skvortsov, ${ }^{1,2, *}$ Tung Tran, ${ }^{3, \dagger}$ and Mirian Tsulaia, ${ }^{4, *}$ \\ ${ }^{1}$ Albert Einstein Institute, Am Mühlenberg 1, D-14476, Potsdam-Golm, Germany \\ ${ }^{2}$ Lebedev Institute of Physics, Leninsky ave. 53, 119991 Moscow, Russia \\ ${ }^{3}$ Arnold Sommerfeld Center for Theoretical Physics, Ludwig Maximilian University of Munich, \\ Theresienstr. 37, D-80333 München, Germany \\ ${ }^{4}$ School of Physics M013, The University of Western Australia, 35 Stirling Highway, Crawley, Perth, WA 6009, Australia
}

(Received 4 May 2018; published 18 July 2018)

\begin{abstract}
An example of a higher spin gravity in four-dimensional flat space has recently been constructed by D. Ponomarev and E. D. Skvortsov, J. Phys. A, 50, 095401(2017). This theory is chiral and the action is written in the light-cone gauge. The theory has certain stringy features, e.g., admits Chan-Paton factors. We show that the theory is consistent, both at the classical and quantum level. Even though the interactions are nontrivial, due to the coupling conspiracy all tree level amplitudes vanish on shell. The loop corrections also vanish. Therefore, the full quantum $S$ matrix is one and the theory is consistent with the numerous no-go theorems. This provides the first example of a (quantum) interacting higher spin gravity with an action. We argue that higher spin gravities in anti-de Sitter space should display the same features.
\end{abstract}

DOI: 10.1103/PhysRevLett.121.031601

Introduction.-Higher spin gravities (HiSGRA) are hypothetical theories that contain graviton and massless fields with spin greater than two. HiSGRAs have a checkered past, since they had long been believed not to exist due to many no-go theorems $[1,2]$, most notably the Weinberg low energy theorem [5] and the ColemanMandula theorem [6]. The theorems directly constrain the $S$ matrix: the former does not allow massless fields with spin greater than two to couple nontrivially to the usual low spin particles, and the latter prevents the $S$ matrix from having symmetry generators that transform as tensors under the Lorentz group.

While the theorems restrict the footprints of interactions at infinity, they have little to say about local effects. Intriguingly, massless higher spin fields were shown to have some consistent local cubic interactions, with the first positive results having been obtained in the light-cone gauge $[7,8]$. One of the greatest advantages of the lightcone approach is that it operates only with physical degrees of freedom (d.o.f.) with the idea of explicitly constructing field dependent realization of the Poincaré algebra.

However, the existence of cubic vertices does not yet guarantee that the higher point amplitudes respect Poincaré symmetries. The analysis of closure of the Poincaré algebra at the quartic order was performed in $[9,10]$ and left some

Published by the American Physical Society under the terms of the Creative Commons Attribution 4.0 International license. Further distribution of this work must maintain attribution to the author(s) and the published article's title, journal citation, and DOI. Funded by SCOAP ${ }^{3}$. possibilities open. Recently in Ref. [1], which is heavily based on Refs. $[9,10]$, it was shown that there exists a simple solution for the Poincaré algebra generators to all orders. The solution was called chiral HiSGRA since its interaction vertices discriminate between helicities: there are more fields with positive helicities than with negative.

Despite its simplicity, the chiral HiSGRA has all the features that HiSGRAs are expected to have on general grounds. The spectrum consists of all massless integer spin fields, starting with the scalar field. The graviton is a part of this spectrum and the theory has a dimensionful coupling constant, which can be identified with the Planck length, $l_{p}$. The theory admits Yang-Mills gaugings [11], which follow the stringy Chan-Paton pattern. All fields have nontrivial (self- and gravitational) interactions that are crucial for classical consistency.

The purpose of this Letter is to report that the chiral HiSGRA is consistent both at the classical and quantum levels. Moreover, it is not in contradiction with the no-go theorems: the couplings conspire in such a way that the full $S$ matrix is 1 and, therefore, when observed at infinity, higher spin fields appear to have trivial scattering. Nevertheless, the chiral HiSGRA provides the first example of a quantum HiSGRA. Moreover, it is the first example of an interacting HiSGRA whose action can explicitly be written. We will also argue that the anti-de Sitter (AdS) HiSGRA counterparts should follow the same pattern, though in a more complicated way.

Chiral higher spin gravity.-Chiral HiSGRA is known in the light-cone gauge and we present the action directly in momentum space to facilitate the computation of amplitudes. The $4 d$ momentum is $\boldsymbol{p}=\left(p^{+}, p^{-}, p, \bar{p}\right)$ and $p^{+}$is 
usually denoted by $\beta$. In the light-cone gauge a massless spin- $s$ field is represented by a pair of scalar fields: $\Phi_{p}^{ \pm s} \equiv \Phi^{ \pm s}(\boldsymbol{p}), \Phi_{p}^{+s^{\dagger}}=\Phi_{-p}^{-s}$. It is also possible and computationally convenient to consider a version of the theory, the "higher spin glue," by taking $\Phi_{s}$ to be $u(N)$ valued. The action of the chiral HiSGRA in momentum space reads

$$
\begin{aligned}
S= & -\sum_{\lambda} \int d^{4} \boldsymbol{p} \operatorname{Tr}\left[\Phi_{\boldsymbol{p}}^{\lambda \dagger} \Phi_{\boldsymbol{p}}^{\lambda}\right] \boldsymbol{p}^{2} \\
& +\sum_{\lambda_{1,2,3}} C_{\lambda_{1}, \lambda_{2}, \lambda_{3}} \int d^{4} \boldsymbol{p}_{1,2,3} \frac{\overline{\mathbb{P}}^{\lambda_{1}+\lambda_{2}+\lambda_{3}}}{\beta_{1}^{\lambda_{1}} \beta_{2}^{\lambda_{2}} \beta_{3}^{\lambda_{3}}} \operatorname{Tr}\left[\Phi_{\boldsymbol{p}_{1}}^{\lambda_{1}} \Phi_{\boldsymbol{p}_{2}}^{\lambda_{2}} \Phi_{p_{3}}^{\lambda_{3}}\right] \\
& \times \delta^{4}\left(\boldsymbol{p}_{1}+\boldsymbol{p}_{2}+\boldsymbol{p}_{3}\right),
\end{aligned}
$$

where $\operatorname{Tr}$ is the trace over the implicit $u(N)$ indices, $\overline{\mathbb{P}} \equiv \overline{\mathbb{P}}_{12} \equiv \bar{p}_{1} \beta_{2}-\bar{p}_{2} \beta_{1}$. It is crucial for the closure of the Poincare algebra to choose the coupling constants as $[1,9,10]$

$$
C_{\lambda_{1}, \lambda_{2}, \lambda_{3}}=\frac{\left(l_{p}\right)^{\lambda_{1}+\lambda_{2}+\lambda_{3}-1}}{\Gamma\left(\lambda_{1}+\lambda_{2}+\lambda_{3}\right)} .
$$

The $\Gamma$-factor requires the sum over helicities in the vertex to be positive and triplets,+++++- , and +-- are present in general. There is a dimensionful coupling constant $l_{p}$ to be associated with the Planck length as the $(+2,+2,-2)$ vertex present here is a part of the usual Einstein-Hilbert action. The light-cone approach is very close to the spinor-helicity formalism, as it was noted, e.g., in Refs. [12-15], and the vertex has a clear interpretation,

$$
\frac{\overline{\mathbb{P}} \lambda_{1}+\lambda_{2}+\lambda_{3}}{\beta_{1}^{\lambda_{1}} \beta_{2}^{\lambda_{2}} \beta_{3}^{\lambda_{3}}} \sim[12]^{\lambda_{1}+\lambda_{2}-\lambda_{3}}[23]^{\lambda_{2}+\lambda_{3}-\lambda_{1}}[13]^{\lambda_{1}+\lambda_{3}-\lambda_{2}} .
$$

Lastly, the presence of the Chan-Paton factors leads to significant simplifications as only color-ordered amplitudes need to be computed.

Tree amplitudes.-We would like to compute all physical $n$-point tree amplitudes, i.e., amplitudes $A_{n}\left(\boldsymbol{p}_{1}, \ldots, \boldsymbol{p}_{n}\right)$ with external legs being on shell, $\boldsymbol{p}_{i}^{2}=0$. It turns out that all $n$-point amplitudes can be computed recursively if lowerorder amplitudes with one external off-shell leg are known:

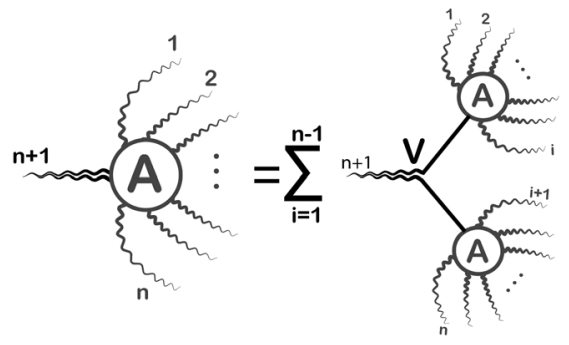

The picture illustrates that the $(n+1)$-point amplitude with one off-shell leg can be obtained as a sum over all ways to attach $(i+1)$ - and $(n+1-i)$-point amplitudes to the cubic vertex. The legs being attached have to be off shell, which explains why we need to know lower-order amplitudes with just one off-shell leg. The simplest amplitude is the 4-point one [16]

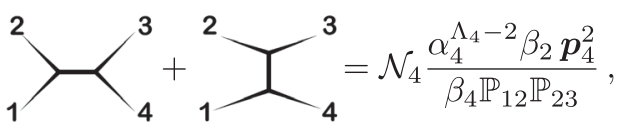

where we define $\Lambda_{n}=\lambda_{1}+\cdots+\lambda_{n}$,

$$
\mathcal{N}_{n}=\frac{(-)^{n}}{2^{n-2} \Gamma\left(\Lambda_{n}-(n-3)\right) \prod_{i=1}^{n} \beta_{i}^{\lambda_{i}-1}},
$$

and $\alpha_{n}=\sum_{i<j}^{n-2} \overline{\mathbb{P}}_{i j}+\overline{\mathbb{P}}_{n-1, n}$, e.g., $\alpha_{4}=\overline{\mathbb{P}}_{12}+\overline{\mathbb{P}}_{34}$.

Thanks to the $\boldsymbol{p}_{4}^{2}$ factor, the physical amplitude $A_{4}$ vanishes. Now it is a matter of direct computation to prove, with the use of Eq. (3), that the $n$-point amplitude is

$$
A_{n}=\mathcal{N}_{n} \frac{\alpha_{n}^{\Lambda_{n}-(n-2)} \beta_{2} \ldots \beta_{n-2} \boldsymbol{p}_{n}^{2}}{\beta_{n} \mathbb{P}_{12} \ldots \mathbb{P}_{n-2, n-1}}
$$

Again, we see that it has $\boldsymbol{p}_{n}^{2}$ factor and therefore the physical $n$-point amplitude $A_{n}$ vanishes. This makes the chiral HiSGRA consistent with the no-go theorems at least at the tree level. It is worth stressing that such a simple result for amplitudes with one off-shell leg relies on the particular form of the coupling constants in Eq. (2). We call this situation coupling conspiracy, since the multitude of nontrivial interactions conspire to cancel in the physical answers. Lastly, vanishing of tree level amplitudes should improve the UV behavior of loop diagrams.

Vacuum loops.-Vacuum diagrams play an important role in the cancellation of legged loop diagrams in the chiral HiSGRA. If we had a covariant action for the theory [18], the one-loop vacuum bubble would be equal to the product of determinants of the kinetic terms [20]

$$
\text { 3: } \quad Z_{1 \text {-loop }}=\frac{1}{\left(z_{0}\right)^{1 / 2}} \prod_{s>0} \frac{\left(z_{s-1}\right)^{1 / 2}}{\left(z_{s}\right)^{1 / 2}},
$$

where $z_{s}=\operatorname{det}_{s, \perp}\left|-\partial^{2}\right|$ is the determinant of $-\partial^{2}$ on the space of transverse and traceless rank- $s$ tensors. The numerator results from the kinetic terms of ghosts. Such determinants have been already studied, both in flat and AdS spaces [21]. The lesson is that the sum (product) over spins needs to be regularized. In our case it is possible [29] to adopt a regularization that makes the cancellation between all numerators and denominators obvious. The same partition function can be interpreted as $Z_{1 \text {-loop }}=$ $\left(z_{0}\right)^{\nu_{0} / 2}, \nu_{0}$ being the total number of d.o.f., which can be regularized as 


$$
\nu_{0}=\sum_{\lambda} 1=1+2 \sum_{s=1}^{\infty} 1=1+2 \zeta(0)=0
$$

i.e., we interpret the spectrum as a scalar field plus 2 d.o.f. per each of $s>0$ field. Then, we find $Z_{1 \text {-loop }}=\left(z_{0}\right)^{\nu_{0} / 2}=1$ [30].

All the other vacuum diagrams vanish without a need to regularize. Indeed, the sum of helicities over all the vertices has to be zero, while for a vertex to contribute the sum over the three ingoing helicities must be positive due to the $\Gamma$ factor in Eq. (2). Therefore, there is always at least one vertex where the $\Gamma$ factor makes the whole diagram vanish.

To summarize, all vacuum diagrams vanish: the one-loop diagram after the appropriate regularization and all the others due to the coupling conspiracy.

Legged loops.-We would like to examine the behavior of legged loop diagrams and see if the coupling conspiracy makes them vanish in one way or another. Two lower order amplitudes are considered in detail and then the general argument is given. We expect the loops not to have any cuts due to the vanishing of tree level amplitudes.

Self energy.-The self-energy diagram, the bubble, is the one we might expect to be UV divergent:

$$
1-\overbrace{\mathbf{k}_{0}}^{\mathbf{q}} \stackrel{2}{2}^{\mathbf{k}_{1}}=\frac{\nu_{0}\left(l_{p}\right)^{\Lambda_{2}-2}}{\Gamma\left[\Lambda_{2}-1\right]} \int \frac{d^{4} q}{(2 \pi)^{4}} \frac{\overline{\mathbb{P}}_{k_{0}-q, p}^{2} \delta_{\Lambda_{2}, 2}}{\left(q-k_{0}\right)^{2}\left(q-k_{1}\right)^{2}},
$$

where we dropped few unessential factors and $k_{0,1}$ are the dual momenta, $\boldsymbol{p}=k_{1}-k_{0}$. We prefer to use the worldsheet-friendly regularization [12,31], which was shown to work nicely in a number of theories, including QCD. The main feature is that $\nu_{0}$ factors out, which allows us to declare vanishing of the whole diagram. Let us, nevertheless, evaluate the integral. For the physical amplitude $\boldsymbol{p}^{2}=0$ the result is simple and finite

$$
\int_{0}^{1} d x\left[x \bar{k}_{0}+(1-x) \bar{k}_{1}\right]^{2}
$$

which is reminiscent of the $\Pi^{++}$amplitude in Ref. [12] for $\Lambda_{2}=2$. If it were not for $\nu_{0}=0$ we would have to add a counterterm to eliminate the correction above since it breaks Lorentz invariance (as discussed for Yang-Mills theory in Ref. [12]).

Vertex correction: The physical three-point amplitude for massless spinning fields is zero for kinematical reasons and we keep one momentum off shell. In the large- $N$ [32] the result is

$$
\underbrace{\mathbf{q}}_{\mathbf{k}_{0}} \sim \nu_{0} \frac{\left(l_{p}\right)^{\Lambda_{3}-3} \overline{\mathbb{P}}_{12}^{\Lambda_{3}}}{\prod_{i=1}^{3} \beta_{i}^{\lambda_{i}} \Gamma\left(\Lambda_{3}-2\right) p_{3}^{2}},
$$

where $\boldsymbol{p}_{3}$ is the off-shell momentum and the expression is similar to the $\Gamma^{+++}$amplitude for QCD [12]. We see that the overall factor $\nu_{0}$ makes it vanish.

General loops: All loop diagrams can be shown to vanish if the total number of d.o.f. is regularized to zero. Indeed, any $l$-loop $n$-point diagram can be represented as the union of elementary sunshine diagrams

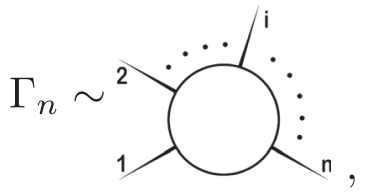

where the external legs may be off shell and can be sewn with other sunshine diagrams. The lower order sunshine diagrams are the self-energy and the vertex correction given above. In general, in the large- $N$ limit for $n>2$ we find an integral of the type

$$
\Gamma_{n} \sim \nu_{0} \frac{1}{\prod_{i=1}^{n} \beta_{i}^{\lambda_{i}}} \int \frac{\mathcal{K}_{n}\left(\overline{\mathbb{P}}_{i j}\right)}{\boldsymbol{p}^{2}\left(\boldsymbol{p}+\boldsymbol{p}_{1}\right)^{2} \ldots\left(\boldsymbol{p}-\boldsymbol{p}_{n}\right)^{2}},
$$

with some numerator $\mathcal{K}_{n}$. What is important is that the "total number of d.o.f." factors out in the form of $\nu_{0}$ [33]. Therefore, each elementary sunshine diagram gets multiplied by zero if we assume Eq. (5), and hence any loop diagram also does so. As a result, all loop diagrams vanish, confirming that $S=1$.

Let us note that the need to regularize the sums over fields is to be expected in any theory with infinitely many fields and represents one of the stringy features of the chiral HiSGRA. Another instance of the same problem arises in dimensional reductions, see, e.g., Ref. [34], and exceptional field theories [35]. In general, it would be helpful to have some worldsheet realization of HiSGRAs exempted from these regularization issues.

Conclusions. - We have studied the simplest chiral HiSGRA with $u(N)$ Chan-Paton factors and showed that it is a fully consistent quantum HiSGRA. Thanks to the coupling conspiracy, both the tree-level amplitudes and the loop corrections vanish. Therefore, $S=1$ to all orders in perturbation theory, which is consistent with the no-go theorems. This provides us with the first example of a quantum HiSGRA. There are several variants of classical chiral HiSGRAs [36] and it would be interesting to see if all of them are quantum consistent.

One may wonder if $S=1$ is a satisfactory answer or whether it means that HiSGRAs are trivial. First, this seems to be the only possible answer consistent with the no-go theorems $[5,6,38]$. Second, pure HiSGRAs are not meant to be realistic models of nature, rather they are toy models of quantum gravity whose importance is perhaps in having the minimal multiplet that allows the graviton to be embedded into a consistent quantum theory. More realistic models should result from matter-coupled and Higgsed HiSGRAs, 
where the solution is expected to be string theory [39]. We argue below that the same reasoning should apply to AdS HiSGRAs whenever they will be constructed and quantized.

Our findings for flat space can also shed some light on AdS HiSGRAs. HiSGRA in AdS [40] are generic duals of free CFT's [48-50]. Indeed, gauge symmetries of massless higher spin fields in AdS translate into dual operators being conserved tensors. The charges associated with the latter signal an extension of the conformal symmetry. The AdS/CFT analog [51-54] of the Coleman-Mandula theorem [6] states that a CFT with a higher spin current is a free one in $d>2$. In any free CFT, say with a free field $\phi$, one can construct infinitely many higher spin currents $J_{s}$ as bilinears $J_{s}=\phi \partial \ldots \partial \phi+\ldots$. The fields of the dual HiSGRA in AdS space are in one-to-one correspondence with $J_{s}$ and bulk interactions should account for nonvanishing $\langle J \ldots J\rangle$, which are built of free partons $\phi$.

Therefore, being dual to a free CFT is a good generalization of the $S=1$ statement from flat space to AdS/CFT holographic $S$ matrix: asymptotic higher spin symmetries in flat space or in AdS imply $S=1$ or $S=$ free CFT, respectively [55]. Based on the analogy above, our conjecture is that AdS HiSGRA should have better UV behavior (compared to the naive power counting) and the systematic reason for the loop corrections to vanish (or be proportional to the tree-level result) should be a factorization of sums over spins (one-loop bubbles), as occurs for the chiral HiSGRA.

Lastly, there are non-HiSGRA examples that share some of the properties of the chiral HiSGRA: self-dual Yang-Mills and self-dual Gravity. They have vanishing tree level amplitudes and finite loop corrections, even though the reasons for cancellation seem to be somewhat different [56,57]. Closer to the chiral HiSGRA are the conformal HiSGRAs [58,59], which are defined in even dimensions as the local part of the induced action of a free CFT in the higher spin background. They should have vanishing tree-level amplitudes and give examples of consistent quantum conformal HiSGRA [60-62]; see also Ref. [63].

We are grateful to Sudarshan Ananth, Nicolas Boulanger, Andrea Campoleoni, Dario Francia, Kirill Krasnov, Ruslan Metsaev, Radu Roiban, Arkady Tseytlin, and especially to Dmitry Ponomarev for very useful discussions. The work of E. S. was supported by the Russian Science Foundation Grant No. 18-72-10123 in association with the Lebedev Physical Institute. The work of T. T. was supported by the DFG Transregional Collaborative Research Centre TRR 33 and the DFG cluster of excellence "Origin and Structure of the Universe." The work of M. T. was supported by the ARC Grant No. DP160103633. *evgeny.skvortsov@aei.mpg.de

†tung.tran@1mu.de

†mirian.tsulaia@gmail.com

[1] D. Ponomarev and E. D. Skvortsov, J. Phys. A 50, 095401 (2017).

[2] An incomplete list includes Refs. [3,4,5] and the review in Ref. [6].

[3] X. Bekaert, N. Boulanger, and S. Leclercq, J. Phys. A 43, 185401 (2010).

[4] S. Weinberg, Phys. Rev. 135, B1049 (1964).

[5] S. R. Coleman and J. Mandula, Phys. Rev. 159, 1251 (1967).

[6] X. Bekaert, N. Boulanger, and P. Sundell, Rev. Mod. Phys. 84, 987 (2012).

[7] A. K. H. Bengtsson, I. Bengtsson, and L. Brink, Nucl. Phys. B227, 41 (1983).

[8] A. K. H. Bengtsson, I. Bengtsson, and L. Brink, Nucl. Phys. B227, 31 (1983).

[9] R. R. Metsaev, Mod. Phys. Lett. A 06, 2411 (1991).

[10] R. R. Metsaev, Mod. Phys. Lett. A 06, 359 (1991).

[11] $o(N)$ color factors were introduced in Ref. [10], but it is easy to see that $u(N)$ and $u s p(N)$ work as well.

[12] D. Chakrabarti, J. Qiu, and C. B. Thorn, Phys. Rev. D 72, 065022 (2005).

[13] S. Ananth, J. High Energy Phys. 11 (2012) 089.

[14] A. K. H. Bengtsson, arXiv:1604.01974.

[15] D. Ponomarev, J. High Energy Phys. 12 (2016) 117.

[16] See also Ref. [1] for the uncolored case while more detail will be given in Ref. [17].

[17] E. Skvortsov, T. Tran, and M. Tsulaia (to be published).

[18] In the covariant description a massless spin- $s$ field is usually represented by a rank- $s$ symmetric tensor and has a gauge symmetry with a rank- $(s-1)$ parameter [19].

[19] C. Fronsdal, Phys. Rev. D 18, 3624 (1978).

[20] Dependence on $N$ is not important for what follows, see also Ref. [17].

[21] For one-loop tests of HiSGRA, see, e.g., Refs. [22-28].

[22] S. Giombi and I. R. Klebanov, J. High Energy Phys. 12 (2013) 068.

[23] S. Giombi, I. R. Klebanov, and Z. M. Tan, Universe 4, 18 (2018).

[24] S. Giombi, I. R. Klebanov, and A. A. Tseytlin, Phys. Rev. D 90, 024048 (2014).

[25] A. Campoleoni, H. A. Gonzalez, B. Oblak, and M. Riegler, J. High Energy Phys. 04 (2016) 034.

[26] M. Günaydin, E. D. Skvortsov, and T. Tran, J. High Energy Phys. 11 (2016) 168.

[27] J.-B. Bae, E. Joung, and S. Lal, J. High Energy Phys. 04 (2016) 061.

[28] E. D. Skvortsov and T. Tran, Universe 3, 61 (2017).

[29] There are many nontrivial one-loop examples that make us confident that the seemingly ad hoc regularization below is correct; see Ref. [30].

[30] M. Beccaria and A. A. Tseytlin, J. Phys. A 48, 275401 (2015).

[31] C. B. Thorn, Nucl. Phys. B699, 427 (2004).

[32] In case of finite $N$ explicit forms of vertex corrections are more complicated. It is important for us that the large- $N$ limit does not affect our general argument that the sum over helicities will factor out, see Ref. [17]. 
[33] It would be interesting to see if the finite expressions for the loop diagrams (modulo the $\nu_{0}$ factor) can be given some interpretation.

[34] E. S. Fradkin and A. A. Tseytlin, Nucl. Phys. B227, 252 (1983).

[35] G. Bossard and A. Kleinschmidt, J. High Energy Phys. 01 (2016) 164.

[36] See Ref. [37]. We also expect SUSY chiral HiSGRA to exist. Likewise, conformal chiral HiSGRA should exist too.

[37] D. Ponomarev, J. High Energy Phys. 12 (2017) 141.

[38] A. Campoleoni, D. Francia, and C. Heissenberg, J. High Energy Phys. 05 (2017) 120.

[39] S. Caron-Huot, Z. Komargodski, A. Sever, and A. Zhiboedov, J. High Energy Phys. 10 (2017) 026.

[40] HiSGRA in AdS is still at its infancy and few terms in the action are known [41,42] or only formally consistent equations are available [43,44]. See Refs. [45-47] for the recent progress in formal HiSGRA.

[41] X. Bekaert, J. Erdmenger, D. Ponomarev, and C. Sleight, J. High Energy Phys. 11 (2015) 149.

[42] C. Sleight and M. Taronna, Phys. Rev. Lett. 116, 181602 (2016).

[43] M. A. Vasiliev, Pisma Zh. Eksp. Teor. Fiz. 51 (1990) 446 [JETP Lett. 51 (1990) 503].

[44] M. A. Vasiliev, Phys. Lett. B 567, 139 (2003).

[45] A. A. Sharapov and E. D. Skvortsov, Nucl. Phys. B921, 538 (2017).

[46] X. Bekaert, M. Grigoriev, and E. D. Skvortsov, Universe 4, 17 (2018).

[47] M. Grigoriev and E. D. Skvortsov, J. High Energy Phys. 05 (2018) 138.

[48] B. Sundborg, Nucl. Phys. B, Proc. Suppl. 102-103, 113 (2001).

[49] E. Sezgin and P. Sundell, Nucl. Phys. B644, 303 (2002).

[50] I. R. Klebanov and A. M. Polyakov, Phys. Lett. B 550, 213 (2002).

[51] J. Maldacena and A. Zhiboedov, J. Phys. A 46, 214011 (2013).
[52] N. Boulanger, D. Ponomarev, E. D. Skvortsov, and M. Taronna, Int. J. Mod. Phys. A 28, 1350162 (2013).

[53] V. Alba and K. Diab, arXiv:1307.8092.

[54] V. Alba and K. Diab, J. High Energy Phys. 03 (2016) 044.

[55] Indeed, both statements are consequences of the linearized gauge symmetry $\delta \Phi_{\mu_{1} \ldots \mu_{s}}=\nabla_{\mu_{1}} \xi_{\mu_{2} \ldots \mu_{s}}+$ perm at the infinity (conformal boundary) of flat (AdS) spaces. See also Ref. [38] for the closely related arguments.

[56] G. Chalmers and W. Siegel, Phys. Rev. D 54, 7628 (1996).

[57] K. Krasnov, Classical Quantum Gravity 34, 095001 (2017).

[58] A. Y. Segal, Nucl. Phys. B664, 59 (2003).

[59] A. A. Tseytlin, Teor. Mat. Fiz. 133, 69 (2002); Theor. Math. Phys. 133, 1376 (2002).

[60] X. Bekaert, E. Joung, and J. Mourad, J. High Energy Phys. 02 (2011) 048.

[61] E. Joung, S. Nakach, and A. A. Tseytlin, J. High Energy Phys. 02 (2016) 125.

[62] M. Beccaria, S. Nakach, and A. A. Tseytlin, J. High Energy Phys. 09 (2016) 034.

[63] A somewhat similar example (vanishing tree-level amplitudes) was constructed in Ref. [64]. Tree level quantum properties of off-shell higher spin "stringlike" systems were considered in Refs. [65-67]. Certain quantum properties of hypothetical HiSGRA in flat space were studied in Ref. [68]. See also Refs. [69,70].

[64] A. Fotopoulos and M. Tsulaia, Phys. Rev. D 76, 025014 (2007).

[65] A. Fotopoulos and M. Tsulaia, J. High Energy Phys. 11 (2010) 086.

[66] M. Taronna, J. High Energy Phys. 04 (2012) 029.

[67] P. Dempster and M. Tsulaia, Nucl. Phys. B865, 353 (2012).

[68] D. Ponomarev and A. A. Tseytlin, J. High Energy Phys. 05 (2016) 184.

[69] R. Roiban and A. A. Tseytlin, J. High Energy Phys. 04 (2017) 139.

[70] M. Taronna, J. High Energy Phys. 05 (2017) 026. 